\title{
Study of power width scaling in scrape-off layer with 2D electrostatic turbulence code based on EAST L-mode discharges
}

Liu, X.; Nielsen, A. H.; Rasmussen, J. J.; Naulin, Volker; Olsen, J.; Xia, T. Y.; Wang, L.; Deng, G. Z.; Wu, X. Q.; Liu, Y.

Total number of authors:

15

Published in:

Physics of Plasmas

Link to article, DOI:

$10.1063 / 1.5083063$

Publication date:

2019

Document Version

Publisher's PDF, also known as Version of record

Link back to DTU Orbit

Citation (APA):

Liu, X., Nielsen, A. H., Rasmussen, J. J., Naulin, V., Olsen, J., Xia, T. Y., Wang, L., Deng, G. Z., Wu, X. Q., Liu, Y., Wang, Y. M., Li, Y. Y., Zhang, Q., Xu, G. S., \& Li, J. (2019). Study of power width scaling in scrape-off layer with 2D electrostatic turbulence code based on EAST L-mode discharges. Physics of Plasmas, 26(4), [042509]. https://doi.org/10.1063/1.5083063

\section{General rights}

Copyright and moral rights for the publications made accessible in the public portal are retained by the authors and/or other copyright owners and it is a condition of accessing publications that users recognise and abide by the legal requirements associated with these rights.

- Users may download and print one copy of any publication from the public portal for the purpose of private study or research.

- You may not further distribute the material or use it for any profit-making activity or commercial gain

- You may freely distribute the URL identifying the publication in the public portal 


\section{Study of power width scaling in scrape-off layer with 2D electrostatic turbulence code based on EAST L-mode discharges}

Cite as: Phys. Plasmas 26, 042509 (2019); https://doi.org/10.1063/1.5083063

Submitted: 27 November 2018 . Accepted: 08 March 2019 . Published Online: 18 April 2019

X. Liu (D), A. H. Nielsen (D), J. J. Rasmussen (D), V. Naulin (D), J. Olsen, T. Y. Xia (D), L. Wang, C. Z. Deng (D) X. Q. Wu, Y. Liu (D), Y. M. Wang (D), Y. Y. Li (D), Q. Zang (D) C. S. Xu, and J. Li

\section{ARTICLES YOU MAY BE INTERESTED IN}

Toward integrated multi-scale pedestal simulations including edge-localized-mode dynamics, evolution of edge-localized-mode cycles, and continuous fluctuations

Physics of Plasmas 23, 055901 (2016); https://doi.org/10.1063/1.4948283

The physics mechanisms of the weakly coherent mode in the Alcator C-Mod Tokamak

Physics of Plasmas 23, 120703 (2016); https://doi.org/10.1063/1.4972088

Self-consistent simulation of transport and turbulence in tokamak edge plasma by coupling SOLPS-ITER and BOUT++

Physics of Plasmas 26, 012508 (2019); https://doi.org/10.1063/1.5084093

\section{Where in the world is AIP Publishing?} Find out where we are exhibiting next 


\title{
Study of power width scaling in scrape-off layer with 2D electrostatic turbulence code based on EAST L-mode discharges
}

Cite as: Phys. Plasmas 26, 042509 (2019); doi: 10.1063/1.5083063

Submitted: 27 November 2018 - Accepted: 8 March 2019 •

Published Online: 18 April 2019

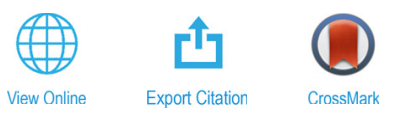

X. Liu, ${ }^{1,2,3,4}$ (D) A. H. Nielsen, ${ }^{2}$ (D) J. J. Rasmussen, ${ }^{2}$ (D) V. Naulin, ${ }^{2}$ (D) J. Olsen, ${ }^{2}$ T. Y. Xia, ${ }^{1}$ (D) L. Wang, ${ }^{1}$ G. Z. Deng, X. Q. Wu, Y. Liu, ' (D) Y. M. Wang, ' (D) Y. Y. Li, ' (D) Q. Zang, ' (D) G. S. Xu, and J. Li, ${ }^{1}$ )

\author{
AFFILIATIONS \\ 'Institute of Plasma Physics, Chinese Academy of Sciences, Hefei 230031, People's Republic of China \\ ${ }^{2}$ Department of Physics, Technical University of Denmark, Kongens Lyngby 2800, Denmark \\ ${ }^{3}$ Sino-Danish College, University of Chinese Academy of Sciences, Beijing 101408, People's Republic of China \\ ${ }^{4}$ Sino-Danish Center for Education and Research, Beijing 101408, People's Republic of China

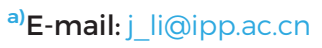

\begin{abstract}
The study of the power width in the scrape-off layer (SOL) is very important for the design and operation of ITER. In this paper, a 2D electrostatic turbulence code is employed to study the power width scaling in L-mode plasmas. It is found that the electron and ion turbulent transports dominate the radial heat fluxes, and the electron heat conduction and the ion heat advection dominate the parallel heat flux in the near and far SOL in L-mode plasmas. The simulated SOL power width agrees well with the Eich scaling [T. Eich et al., Nucl. Fusion 53, 093031 (2013)] and the predictions by the heuristic drift-based model [R. J. Goldston, Nucl. Fusion 52, 013009 (2012)] for selected EAST L-mode discharges. A numerical scaling has been performed based on one of these discharges. The scaling dependence on the safety factor is consistent with the Eich scaling and the scaling exponent of the edge electron temperature is close to that in the ASDEX-Upgrade L-mode scaling [B. Sieglin et al., Plasma Phys. Controlled Fusion 58, 055015 (2016)]. The investigation of the obtained numerical scaling for L-mode plasmas reveals that the SOL power width is influenced by the safety factor, the edge electron density, and the edge electron temperature through the parallel heat transports, the radial turbulent heat transports, and both the parallel and radial heat transports, respectively. The formulation of the turbulence model suggests that the scaling dependence on the poloidal magnetic field (or the plasma current) for the experimental scalings is essentially the scaling dependence on the ballooning length, $q_{95} R$. Based on this idea, a further numerical scaling gives $\lambda_{q} \propto q_{95}^{1.30} B_{t}^{-0.33} R^{1.32} \propto B_{t}^{-0.29}\left(q_{95} R\right)^{1.33}$, which has a strong scaling dependence on the major radius that is different from the Eich scaling.
\end{abstract}

Published under license by AIP Publishing. https://doi.org/10.1063/1.5083063

\section{INTRODUCTION}

The prediction of the divertor heat load is one of the most important issues for the operations of ITER or future fusion devices. The divertor wetted area is a key parameter to characterize the heat load on divertor targets and is dependent on both the power distribution in the scrape-off layer (SOL) and the divertor geometry. To get a predictable scaling law, the SOL power width (or power fall-off width) $\lambda_{q}$ at the outer midplane (OMP) is normally used to avoid the influence from the divertor geometry. A number of experimental studies have been carried out in the last twenty years to scale $\lambda_{q}{ }^{1-10}$ Some of the most prominent work in this area is done by Eich et al. ${ }^{2,4}$ They proposed a multimachine (Eich) scaling law of $\lambda_{q}$ based on the multimachine databases, which predicts that $\lambda_{q, I T E R} \approx 1 \mathrm{~mm}$ for the ITER baseline inductive H-mode scenario. ${ }^{4}$ The Eich scaling of $\lambda_{q}$ shows a strong negative dependence on the poloidal magnetic field at the OMP separatrix $B_{p}$, given by $\lambda_{q}=0.63 B_{p}^{-1.19}$, and is independent of the major radius. ${ }^{4}$ Recently, the range of $B_{p}$ in this scaling is further extended up to about $1.3 \mathrm{~T}$ with C-Mod data. ${ }^{9}$ In EAST, $\lambda_{q}$ scalings also exhibit the same dependence on $B_{p}$, but with about two times larger (the same) amplitudes at the outer (inner) divertor. ${ }^{5,6,8,10}$ The reason for the larger amplitude at the outer divertor is probably due to the lower hybrid wave (LHW) heating scheme. ${ }^{8,11}$

Even though the scaling dependence on $B_{p}$ is robust, recent studies indicate that there is another important scaling parameter that is not included in the Eich scaling, i.e., the edge electron temperature $T_{e, \text { edge. }}{ }^{7,10}$ In ASDEX-Upgrade (AUG) L-mode plasmas, $\lambda_{q}$ scaling 
yields $\lambda_{q} \propto\left(W_{M H D} / n_{e, e \text { dge }}\right)^{-0.93} \propto T_{e, \text { edge }}^{-0.93}$ for stiff pressure profiles, where $n_{e, e d g e}$ is the edge electron density. In EAST H-mode plasmas, the scaling of particle flux fall-off width $\lambda_{j s}$ at the inner divertor is $\lambda_{j s} \propto\left(W_{M H D} / \bar{n}_{e}\right)^{-0.5} \propto T_{e, \text { edge }}^{-0.5}$ for stiff pressure profiles, where $\bar{n}_{e}$ is the line-averaged electron density. ${ }^{10}$ According to Refs. 5 and 6, the ratio of $\lambda_{j s}$ to $\lambda_{q}$ is a constant $(\approx 1.3)$ for measurement by the divertor Langmuir probes. Then, the scaling in EAST H-mode plasmas becomes $\lambda_{q} \propto T_{e . e d g e}^{-0.5}$, which shows a weaker temperature dependence compared with the AUG L-mode scaling. In C-Mod L-, I- and Hmode plasmas, a unified scaling is found, i.e., $\lambda_{q} \propto \bar{p}^{-0.5} \propto W_{M H D}^{-0.5}$, where $\bar{p}$ is the volume-averaged plasma pressure. Although the plasma density is not included to tell whether there is a scaling dependence on $T_{e, \text { edge }}$ or not in C-Mod, the exponent of $W_{M H D}$ in the scaling is the same as that in the EAST H-mode scaling.

There are several theoretical studies ${ }^{12-14}$ on $\lambda_{q}$ scaling, and a heuristic drift (HD)-based model is very successful in explaining the Eich scaling. ${ }^{4,14}$ In the heuristic drift-based model, the ion magnetic drift determines the SOL particle width on the order of the ion gyroradius. ${ }^{14}$ The six-field two-fluid $3 \mathrm{D}$ turbulence module ${ }^{15}$ in the BOUT ++ framework $^{16}$ has reproduced the inverse scaling dependence on the plasma current in both EAST ${ }^{17}$ and C-Mod ${ }^{18} \mathrm{H}$-mode plasmas. In the EAST simulations, the neoclassical and turbulent transports instead of the magnetic drift are found to play more important roles in contributing to the divertor heat flux. ${ }^{17}$ The $3 \mathrm{D}$ gyrokinetic code, XGC1, was also employed to simulate $\lambda_{q}$ and showed consistent results with the Eich scaling. ${ }^{19}$ However, the code predicts a much larger $\lambda_{q}$ for ITER $\left(\lambda_{q, I T E R}=5.9 \mathrm{~mm}\right)$, which is explained to be that the divertor heat flux is dominated by the electron blobby transport in ITER and radial blobby turbulence spreads further because of the wider $\mathrm{E} \times \mathrm{B}$ shear flow layer as a consequence of the larger major radius. ${ }^{19}$ Some of the studies ${ }^{13,20,21}$ employ the transport code, SOLPS, to investigate $\lambda_{q}$, and the study of EAST L-mode plasmas suggests that the parallel heat transports in the SOL contribute more to the inverse scaling dependence on the poloidal magnetic field. ${ }^{21}$

Besides these 3D codes, the 2D turbulence code, HESEL (hot edge-SOL electrostatic), has also been utilized to reproduce the AUG L-mode scaling [no temperature dependence, see Eq. (15) in Ref. 7]. ${ }^{7,22}$ Benefiting from the first-principles perpendicular transports and the low computation time of the HESEL model, a numerical scaling that is dependent on the safety factor at the LCFS $q_{95}$, the electron density at the last closed flux surface (LCFS) $n_{e, L C F S}$, and the electron temperature at the LCFS $T_{e, L C F S}$ has been proposed for L-mode plasmas $^{22}$

$$
\lambda_{q}=(4.74 \pm 0.92) q_{95}^{0.86 \pm 0.05} T_{e, L C F S}^{-0.40 \pm 0.08} n_{e, L C F S}^{0.24 \pm 0.05} .
$$

However, there is no further physical interpretation of this numerical scaling, the neoclassical diffusion terms are implemented without full variations of the electron density and the electron and ion temperatures, and the ion viscous heating is also not implemented. In this paper, we use the newly reimplemented HESEL code (within the BOUT ++ framework $^{16}$ ) with full variations retained in the neoclassical collision terms to study $\lambda_{q}$ scaling based on EAST L-mode discharges. The rest of this paper is organized as follows: Sec. II introduces the formulation of the HESEL model. Section III introduces the processing of the experimental profiles used for the simulations. Section IV shows and discusses the simulation results. The conclusion is drawn in Sec. V.

\section{THE HESEL MODEL}

The HESEL model is a drift-ordered, 2D interchange driven turbulence model based on the Braginskii fluid equations with the selfconsistent neoclassical collisions retained. The model is tailored to simulate interchange turbulence at the OMP in a tokamak. ${ }^{23}$ Figure 1 shows the HESEL simulation domain in the BOUT ++ coordinate system ( $\mathrm{x}, \mathrm{y}$, and $\mathrm{z}$ are in the radial, toroidal, and poloidal directions, respectively), which covers both the closed (the edge region) and open (the SOL and wall regions) field lines. Normally, the inner half of the edge region is set to be the profile forcing region, where the electron density and the electron and ion pressures are forced to align with their initial profiles in a time scale of $\tau_{f}$. In the wall region, the parallel damping is enhanced to avoid blobs hitting the outer radial boundary. The boundary conditions in the radial direction for the inner and outer boundaries are Dirichlet and Neumann, respectively, and the boundary condition in the poloidal direction is periodic.

The HESEL model evolves the electron density $n$, the electron pressure $p_{e}$, the ion pressure $p_{i}$, and the generalized vorticity $w^{*}$. The gyro-Bohm normalization is applied, i.e., $\omega_{c i 0} t \rightarrow t, x / \rho_{s 0} \rightarrow x, n / n_{0}$ $\rightarrow n, T_{e(i)} / T_{e 0} \rightarrow T_{e(i)}, e \varphi / T_{e 0} \rightarrow \varphi$, where $\omega_{c i 0}=e B_{0} / m_{i}$ is the ion gyro-frequency, $\rho_{s 0}=\left(T_{e 0} / m_{i}\right)^{1 / 2} / \omega_{c i 0}$ is the cold ion hybrid thermal gyro-radius, and $e$ is the elementary charge. Here, $B_{0}, n_{0}$, and $T_{e 0}$ are the reference values for the toroidal magnetic field, the electron density, and the electron temperature, all taken at the LCFS from their corresponding initial profiles. With the Boussinesq approximation applied for the polarization term, the equations $\operatorname{are}^{23}$

$$
\begin{aligned}
& \frac{\partial}{\partial t} n+\frac{1}{B}\{\varphi, n\}+n \kappa(\varphi)-\kappa\left(p_{e}\right)=\Lambda_{n}, \\
& \frac{\partial}{\partial t} w^{*}+\left\{\varphi, w^{*}\right\}+\left\{\partial_{x} \varphi, \partial_{x} p_{i}\right\}+\left\{\partial_{z} \varphi, \partial_{z} p_{i}\right\}-\kappa\left(p_{e}+p_{i}\right)=\Lambda_{w^{*}}, \\
& \frac{3}{2} \frac{\partial}{\partial t} p_{e}+\frac{3}{2} \frac{1}{B}\left\{\varphi, p_{e}\right\}+\frac{5}{2} p_{e} \kappa(\varphi)-\frac{5}{2} \kappa\left(p_{e} T_{e}\right)=\Lambda_{p_{e}}, \\
& \frac{3}{2} \frac{\partial}{\partial t} p_{i}+\frac{3}{2} \frac{1}{B}\left\{\varphi, p_{i}\right\}+\frac{5}{2} p_{i} \kappa(\varphi)+\frac{5}{2} \kappa\left(p_{i} T_{i}\right)-p_{i} \kappa\left(p_{e}+p_{i}\right)=\Lambda_{p_{i}},
\end{aligned}
$$

where $w^{*}=\nabla_{\perp}^{2} \varphi^{*}$ and $\varphi^{*}=\varphi+p_{i}$ is the generalized potential, $\{f, g\}=\hat{b} \times \nabla \mathrm{f} \cdot \nabla g$ is the Poisson bracket operator, and $\kappa(f)$

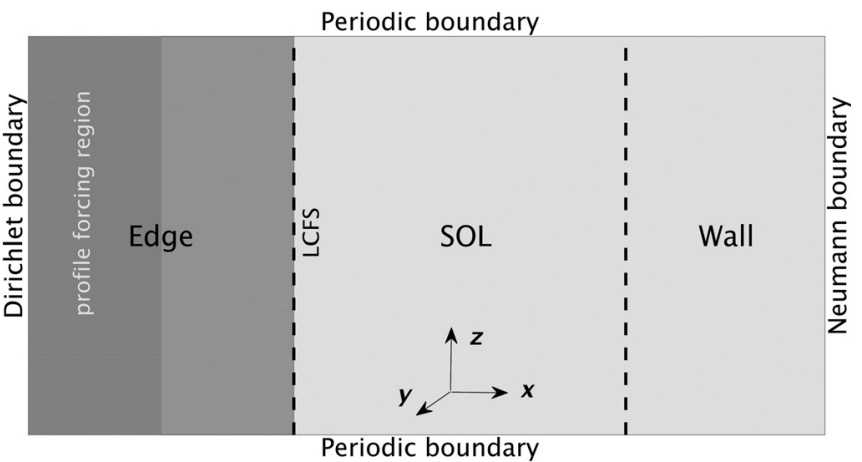

FIG. 1. The simulation domain and the boundary conditions of the HESEL model in the BOUT ++ coordinate system. $x, y$, and $z$ are in the radial, toroidal, and poloidal directions, respectively ( $y$ and $z$ are in the poloidal and toroidal directions, respectively, for the old implementation of HESEL in Ref. 24). 
$=\nabla \times(\hat{b} / B) \cdot \nabla f$ is the magnetic curvature operator. $\hat{b}=\vec{B} / B$ is the base vector along the magnetic field lines. In a Cartisian left-handed coordinate system (see Fig. 1), the explicit forms of $\{f, g\}$ and $\kappa(f)$ are $\partial_{z} f \partial_{x} g-\partial_{x} f \partial_{z} g$ and $\rho_{s 0} \partial_{z} f /(R+a)$, respectively, where $R$ and $a$ are the major and minor radii. The perpendicular diffusion terms and the parallel damping terms on the right-hand side of Eq. (2) $\operatorname{are}^{23,24}$

$$
\begin{aligned}
\Lambda_{n}= & C_{n}-\frac{n}{\tau_{n}} \sigma_{\text {open }}-\Im \sigma_{\text {closed }}+\sigma_{\text {force }} \frac{n_{\text {init }}-n}{\tau_{f}}, \\
\Lambda_{w^{*}}= & \frac{3}{10} D_{i 0} \nabla_{\perp}^{2} w^{*}-\left(\frac{w^{*}}{\tau_{n}}-S\right) \sigma_{\text {open }}-\mathfrak{\Im} \sigma_{\text {closed }}, \\
\Lambda_{p_{e}}= & C_{p_{e}}-\left(\frac{T_{e}^{7 / 2}}{\tau_{\text {sh }, e}}+\frac{9}{2} \frac{p_{e}}{\tau_{n}}\right) \sigma_{\text {open }}-3.21 \bar{T}_{e} \Im \sigma_{\text {closed }} \\
& +\sigma_{\text {force }} \frac{p_{e, i n i t}-p_{e}}{\tau_{f}}-Q_{\Delta}-Q_{R}, \\
\Lambda_{p_{i}}= & C_{p_{i}}-\left(\frac{T_{i}^{7 / 2}}{\tau_{s h, i}}+\frac{9}{2} \frac{p_{i}}{\tau_{n}}-p_{i} S\right) \sigma_{o p e n}-\bar{p}_{i} \Im \sigma_{\text {closed }}+\sigma_{\text {force }} \frac{p_{i, \text { init }}-p_{i}}{\tau_{f}} \\
& +\eta\left[4\left(\partial_{x z}^{2} \varphi^{*}\right)^{2}+\left(\partial_{x x}^{2} \varphi^{*}-\partial_{z z}^{2} \varphi^{*}\right)^{2}\right]+Q_{\Delta}+Q_{R},
\end{aligned}
$$

where $n_{\text {init }}, p_{e, \text { init }}$, and $p_{i \text {,init }}$ are the initial profiles, and $\sigma_{\text {open }}$, $\sigma_{\text {closed }}$, and $\sigma_{\text {force }}$ are the step functions in the wall and SOL, the edge, and the profile forcing regions, respectively, i.e.,

$$
\begin{gathered}
\sigma_{\text {open }}=\frac{1}{2}\left[1+\tanh \left(\frac{x-x_{L C F S}}{\delta_{s}}\right)\right], \\
\sigma_{\text {closed }}=\frac{1}{2}\left[1-\tanh \left(\frac{x-x_{L C F S}}{\delta_{s}}\right)\right], \\
\sigma_{\text {force }}=\frac{1}{2}\left[1-\tanh \left(\frac{x-x_{\text {force }}}{\delta_{s}}\right)\right] .
\end{gathered}
$$

The parallel dynamics is parametrized as sinks. On the open field lines, the damping times for the density advection and the electron and ion Spitzer-Härm heat conduction are

$$
\tau_{n}=\frac{L_{b}}{2 M c_{s}} \omega_{c i 0}, \quad \tau_{s h, e}=\frac{m_{e} \nu_{e i 0} L_{b}^{2}}{3.16 T_{e 0}} \omega_{c i 0}, \quad \tau_{s h, i}=\frac{m_{i} \nu_{i i 0} L_{b}^{2}}{3.9 T_{i 0}} \omega_{c i 0},
$$

where $c_{s}=\sqrt{\left(T_{e}+T_{i}\right) / m_{i}}$ is the hot ion sound speed, $L_{b} \approx q R$ ( $q$ is the safety factor at the LCFS; since the safety factor at the LCFS is not available experimentally, the safety factor at $95 \%$ of the poloidal magnetic flux $q_{95}$ is used) is the ballooning length with the assumption that blobs are generated at the OMP with a $60^{\circ}$ poloidal extend, ${ }^{23} M$ is the Mach number (normally is assumed to be 0.5), and $\nu_{e i 0}$ and $\nu_{i i 0}$ are the reference electron-ion and ion-ion collision frequencies, respectively. The collisional limits for the electron and ion SpitzerHärm heat conduction are $\nu_{e}^{*} \equiv \frac{L_{b} \nu_{\text {eii }}}{v_{\text {the }}} \geq 1$ and $\nu_{i}^{*} \equiv \frac{L_{b} \nu_{i i 0}}{v_{\text {thi }}} \geq 1$, respectively, where $v_{\text {the } 0}$ and $v_{\text {thi } 0}$ are the reference electron and ion thermal velocities. ${ }^{13}$ For all the simulations in this paper, $\nu_{e}^{*} \geq 8.3$ and $\nu_{i}^{*} \geq 2.5$, satisfying the premise of the Spitzer-Härm conduction.

The compression of the parallel current on the open field lines is expressed as the sheath damping term ${ }^{24}$

$$
S=\frac{c_{s}}{L_{c} \omega_{c i 0}}\left[1-\exp \left(\varphi_{m}-\frac{\varphi}{T_{e}}\right)\right]
$$

where $\varphi_{m}=\ln \left(\sqrt{m_{i} /\left(2 \pi m_{e}\right)}\right)$ is the Bohm potential and $L_{c}$ is the parallel connection length (typical value for EAST is $15 \mathrm{~m}$ ). ${ }^{24}$ While on the closed field lines, the parallel current compression is approximated as the drift wave term ${ }^{24}$

$$
\mathfrak{I}=\alpha_{d w}\left(\tilde{T}_{e}+\frac{\bar{T}_{e}}{\bar{n}} \tilde{n}-\tilde{\varphi}\right),
$$

where $\tilde{f}=f-\bar{f}, \bar{f}=\frac{1}{L_{z}} \int_{0}^{L_{z}} f d z$ is the poloidal average operator and the coefficient $\alpha_{d w}$ is defined as

$$
\alpha_{d w}=\frac{2 T_{e 0}}{m_{e} \nu_{e i 0} L_{b}^{2}} \frac{1}{\omega_{c i 0}} .
$$

For the perpendicular collisional diffusion, the reference electron and ion diffusion coefficients with the neoclassical correction are ${ }^{23,25}$

$$
\begin{aligned}
& D_{e 0}=\left(1+\frac{R}{a} q^{2}\right) \frac{\rho_{e 0}^{2} \nu_{e i 0}}{\rho_{s 0}^{2} \omega_{c i 0}}, \\
& D_{i 0}=\left(1+\frac{R}{a} q^{2}\right) \frac{\rho_{i 0}^{2} \nu_{i i 0}}{\rho_{s 0}^{2} \omega_{c i 0}},
\end{aligned}
$$

where $\rho_{e 0}$ and $\rho_{i 0}$ are the reference electron and ion gyro-radii, and $q_{95}$ is used to replace the safety factor at the LCFS. One should be aware of the fact that the neoclassical correction in principle should only apply to the poloidal averaging profiles on the closed field lines, not the turbulent fluctuations, since the time scale of the turbulent fluctuations is much smaller than that of the neoclassical diffusions. ${ }^{25}$ In the HESEL model, the neoclassical correction has been applied to the full fields (meaning that the dissipation of the turbulent fluctuations is largely overestimated) on both the closed and open field lines to simplify the implementation and to avoid the numerical instability. The neoclassical collision terms with full variations are ${ }^{23}$

$$
\left.\begin{array}{rl}
C_{n} & =-\nabla \cdot\left(n \vec{u}_{R}\right) \\
C_{p_{e}} & =-\nabla \cdot\left(p_{e} \vec{u}_{R}\right)+\frac{29}{12} \nabla \cdot\left(D_{e} n \nabla_{\perp} T_{e}\right) \\
C_{p_{i}} & =-\frac{5}{2} \nabla \cdot\left(p_{i} \vec{u}_{R}\right)+2 \nabla \cdot\left(D_{i} n \nabla_{\perp} T_{i}\right) \\
\eta & =\frac{3}{10} n D_{i}
\end{array}\right\} \text { Full Collisional Model, }
$$

where the electron and ion diffusion coefficients and the resistive drift velocity are

$$
\begin{aligned}
& D_{e}=D_{e 0} n T_{e}^{-1 / 2} B^{-2}, \\
& D_{i}=D_{i 0} n T_{i}^{-1 / 2} B^{-2},
\end{aligned}
$$

and

$$
\vec{u}_{R}=-\frac{D_{e}}{T_{e}}\left[\left(T_{e}+T_{i}\right) \nabla_{\perp} n / n+\left(\nabla_{\perp} T_{i}-\frac{1}{2} \nabla_{\perp} T_{e}\right)\right],
$$

respectively. A standard collisional model (partially linearized) is obtained by neglecting the variation of the electron and ion diffusion coefficients in Eqs. (11) and (12) and the second term inside the square brackets in Eq. (12). The standard collisional model is written as 


$$
\left.\begin{array}{rl}
C_{n} & =D_{n 0} \nabla_{\perp}^{2} n \\
C_{p_{e}} & =D_{n 0} \nabla \cdot\left(T_{e} \nabla_{\perp} n\right)+\frac{29}{12} D_{e 0} \nabla \cdot\left(n \nabla_{\perp} T_{e}\right) \\
C_{p_{i}} & =\frac{5}{2} D_{n 0} \nabla \cdot\left(T_{i} \nabla_{\perp} n\right)+2 D_{i 0} \nabla \cdot\left(n \nabla_{\perp} T_{i}\right) \\
\vec{u}_{R} & =-\left(D_{n 0} \nabla_{\perp} n\right) / n \\
\eta & =\frac{3}{10} n D_{i 0}
\end{array}\right\} \text { Standard Collisional Model, }
$$

where $D_{n 0}=\left(1+T_{i 0} / T_{e 0}\right) D_{e 0}$. The energy between the electron and ion is exchanged through the resistive drift term $Q_{R}$ and the elastic electron-ion collision term $Q_{\Delta}{ }^{23}$

$$
\begin{aligned}
Q_{R} & =\vec{u}_{R} \cdot \nabla_{\perp} p_{i}, \\
Q_{\Delta} & =\frac{3 m_{e} \omega_{c i 0}}{m_{i} v_{e i}}\left(p_{e}-p_{i}\right) .
\end{aligned}
$$

The HESEL model with the standard collisional model has already been implemented in Ref. 24 based on the previous ESEL ${ }^{26}$ code. Benefiting from the high flexibility and usability of the BOUT ++ framework, ${ }^{16}$ the model is reimplemented with both the standard and full collisional models included. Since the neoclassical transport is reported to be important in previous studies, ${ }^{17,19}$ the full collisional model is employed to keep all collisional effects in this paper to study the SOL power width.

\section{SETUP OF THE SIMULATIONS}

Since the poloidal magnetic field is the most significant scaling parameter in the power width scalings, ${ }^{4,9}$ three L-mode discharges in EAST are selected for the simulations, where mainly the plasma current varies. The main plasma parameters, such as the plasma current $I_{p}$, the poloidal magnetic field at the LCFS at the OMP $B_{p}$, the toroidal magnetic field at the magnetic axis $B_{t}$, the safety factor at $95 \%$ of the poloidal magnetic flux $q_{95}$, the major radius $R$, the minor radius $a$, and the power entering SOL $P_{S O L}$, are listed in Table I. All three discharges were configured with upper single null ( $d$ Rsep $\approx 22 \mathrm{~mm}$, the upper triangularity ranges from 0.53 to 0.61 , and the plasma elongation is around 1.6), heated by neutral beam injection (NBI), and fueled by supersonic molecule beam injection. The divertor was in the attached condition, the wall was coated by lithium, and the toroidal magnetic field was configured to the forward direction (ion $\mathrm{B} \times \nabla \mathrm{B}$-drift directed downward).

The measured electron density and the electron and ion temperature profiles are fitted before being used for the simulations. The electron density was measured by reflectometry (Refl). ${ }^{27-29}$ The electron temperature was measured by a Michelson interferometer (MI), ${ }^{30}$ a heterodyne radiometer system (HRS), ${ }^{31}$ and Thomson scattering (TS). ${ }^{32}$ The ion temperature was measured by charge exchange recombination spectroscopy (CXRS). ${ }^{33}$ Figure 2 demonstrates the fits of the measured profiles for shot \#71598. In the far SOL, background paddings (the padding values for the plasma density and temperatures are $1.0 \times 10^{17} \mathrm{~m}^{-3}$ and $1 \mathrm{eV}$, respectively) are added to force the fitted profiles to align with the backgrounds. For the ion temperature measurements, the fitting coefficients from the fitted electron temperature profile except for the amplitude are applied for the fit of the ion temperature profile, meaning that the shape of the fitted ion temperature profile is the same as that of the fitted electron temperature profile. The reason to match the ion temperature profile with the electron temperature profile is due to that there are no data near the LCFS [see Fig. 2(c)] and the measurement uncertainties are large for the CXRS measurements. The location of the LCFS is determined by the EFIT code and is used to evaluate the reference electron density $n_{0}$, the reference electron temperature $T_{e 0}$, and the reference ion temperature $T_{i 0}$ at the LCFS as the input parameters (see Table I).

The fitted profiles in the shaded areas of Fig. 2 are used to generate initial profiles. Figure 3 shows the initial profiles for all three discharges. As the plasma current increases, we see that the gradients of the electron and ion pressures also increase in the edge region. The radial and poloidal lengths of the simulation region for the three discharges are $240 \mathrm{~mm}$ and $120 \mathrm{~mm}$, respectively, and the corresponding grid sizes are 768 and 384 in $\mathrm{x}$ - and $\mathrm{z}$-axes, respectively. The values of the normalization factor of space $\rho_{s 0}$ and the radial grid spacing $d x$ (the poloidal grid spacing is equal to $d x$ ) are listed in Table I.

\section{SIMULATION RESULTS AND DISCUSSION}

\section{A. Parallel and radial heat fluxes}

The parallel heat fluxes in the HESEL model are evaluated as

$$
\begin{aligned}
q_{/ /, e, a d} & =\left\langle 9 p_{e} M c_{s}\right\rangle_{z}, \\
q_{/ /, i, a d} & =\left\langle 9 p_{i} M c_{s}\right\rangle_{z},
\end{aligned}
$$

for the electron and ion heat advections, respectively, and as

$$
\begin{aligned}
& q_{/ /, e, s h}=\left\langle n_{0} T_{e 0}^{-5 / 2} \frac{T_{e}^{7 / 2}}{\tau_{s h, e} / \omega_{c i 0}} L_{b}\right\rangle_{z}, \\
& q_{/ /, i, s h}=\left\langle n_{0} T_{i 0}^{-5 / 2} \frac{T_{i}^{7 / 2}}{\tau_{s h, i} / \omega_{c i 0}} L_{b}\right\rangle z,
\end{aligned}
$$

for the electron and ion Spitzer-Härm heat conduction, respectively, where $\langle f\rangle_{z}=\bar{f}$ is the poloidal average operator. The radial electron and ion neoclassical heat fluxes for the full collisional model are

$$
\begin{aligned}
q_{r, e, \text { nеoс }} & =\left\langle p_{e} u_{R, x}-\frac{29}{12} D_{e} n \partial_{x} T_{e}\right\rangle_{z}, \\
q_{r, i, \text { neoc }} & =\left\langle\frac{5}{2} p_{i} u_{R, x}-2 D_{i} n \partial_{x} T_{i}\right\rangle_{z},
\end{aligned}
$$

where $u_{R, x}$ is the radial component of the resistive drift velocity. The radial electron and ion $\mathrm{E} \times \mathrm{B}$ turbulent heat fluxes are defined as 
TABLE I. The main parameters for the selected EAST L-mode discharges. The parameters marked in bold are used as input parameters for the simulations.

\begin{tabular}{lcccccccccccc}
\hline \hline & $I_{p}(\mathrm{kA})$ & $B_{p}(\mathrm{~T})$ & $\boldsymbol{B}_{\boldsymbol{t}}(\mathrm{T})$ & $\boldsymbol{q}_{\mathbf{9 5}}$ & $\boldsymbol{T}_{\boldsymbol{e} \mathbf{0}}(\mathrm{eV})$ & $\boldsymbol{T}_{\boldsymbol{i} \mathbf{0}}(\mathrm{eV})$ & $\boldsymbol{n}_{\mathbf{0}}\left(10^{19} \mathrm{~m}^{-3}\right)$ & $\boldsymbol{R}(\mathrm{m})$ & $\boldsymbol{a}(\mathrm{m})$ & $P_{S O L}(\mathrm{MW})$ & $\rho_{s 0}(\mathrm{~mm})$ & $d x$ \\
\hline \#71598 at 3.3 s & 400 & 0.159 & -2.43 & 6.70 & 33.3 & 50.1 & 1.69 & 1.87 & 0.42 & 0.99 & 0.42 & 0.74 \\
\#80768 at 3.8 s & 450 & 0.180 & -2.34 & 5.82 & 38.1 & 49.6 & 1.72 & 1.88 & 0.42 & 1.24 & 0.47 & 0.67 \\
\#71597 at 3.3 s & 500 & 0.192 & -2.43 & 5.46 & 39.4 & 48.5 & 1.74 & 1.87 & 0.43 & 1.02 & 0.46 & 0.68 \\
\hline \hline
\end{tabular}

$$
\begin{aligned}
q_{r, e, t u r b} & =\left\langle\frac{3}{2} p_{e} u_{E, x}\right\rangle_{z}, \\
q_{r, i, t u r b} & =\left\langle\frac{3}{2} p_{i} u_{E, x}\right\rangle_{z},
\end{aligned}
$$

where $u_{E, x}$ is the $\mathrm{E} \times \mathrm{B}$ drift velocity in the radial direction. The radial heat flux contributed by the ion viscous heating term [the fifth term of the ion pressure equation in Eq. (3)] is hard to write explicitly and, thus, is not calculated.

The time-averaged parallel and radial heat fluxes in the saturation phase for the simulations are plotted in Fig. 4. From the first row, we learn that the electron heat conduction and the ion heat advection dominate the parallel heat flux in the near and far SOL for all three discharges, respectively. Since the damping rate of the electron heat conduction is much faster than that of the ion heat advection in the near SOL and the electron heat conduction also has much stronger temperature dependence than that for the ion heat advection, the heat flux profile of the electron heat conduction is much narrower than that of the ion heat advection. The shape of the heat flux profiles between the electron and ion heat advections is similar because the ion temperature is highly coupled with the electron temperature through the electronion heat exchanges [see Eq. (14)] in high collisional plasmas. As the plasma current increases, the profiles of the parallel heat fluxes become narrower for both the heat conduction and the heat advections.

The second row of Fig. 4 shows the distributions of the radial heat fluxes. In the profile forcing region (the shaded areas), the turbulent heat fluxes are zero because there are no fluctuations in this region. The electron neoclassical heat flux is negligible compared with the ion neoclassical heat flux in the whole simulation region. The electron and ion turbulent heat fluxes are both significant in the edge and SOL regions, and the ion neoclassical heat flux is relatively small compared with the turbulent heat fluxes. Compared with the lowcurrent case (shot \#71598), the turbulent heat fluxes are smaller in the high-current case (shot \#71597) because of the faster parallel damping in the SOL. The medium-current case (shot \#80768) has similar instead of smaller turbulent heat fluxes with the low-current case due to its higher temperature gradients (see Fig. 3) and smaller toroidal magnetic field (see Table I), resulting in larger interchange instability. The ion neoclassical heat flux near the profile forcing region drops dramatically because the gradient of the ion temperature profile near the profile forcing region is smaller compared with that in the profile forcing region (see the time-averaged profiles in Fig. 5). It should be noted that the sum of four kinds of radial heat fluxes in Fig. 4 (black solid lines) is not well conserved in the edge region (the forcing region is not included) because the radial heat flux from the viscous heating term is not counted. In the repeated simulations where the viscous heating term is turned off, it is conserved in this region.

\section{B. Simulated SOL power width}

In this paper, the integrated power width $\left(\lambda_{\text {int }} \equiv \int q d x / q_{L C F S}\right.$, where $q_{L C F S}$ is the parallel heat flux at the LCFS) ${ }^{4}$ is used to evaluate the SOL power width at the OMP, which avoids fitting uncertainties introduced by the exponential fit. Figure 6 shows the evolution of the parallel electron heat flux $\left(q_{e}=q_{/ /, e, s h}+q_{/ /, e, a d}\right)$ width $\lambda_{\text {qe }}$, the parallel ion heat flux $\left(q_{i}=q_{/ /, i, s h}+q_{/ /, i, a d}\right)$ width $\lambda_{q i}$, and the total parallel heat flux $\left(q=q_{e}+q_{i}\right)$ width $\lambda_{q}$. The mean values and the standard deviations of the data in the shaded areas are displayed in the bottom of each panel with the same color. Clearly, $\lambda_{q e}, \lambda_{q i}$, and $\lambda_{q}$ decrease as the poloidal magnetic field or the plasma current increases.

In Fig. 7, the simulated SOL power widths are compared with the Eich scaling, ${ }^{4}$ the EAST L-mode scaling, ${ }^{6}$ and the predictions by the
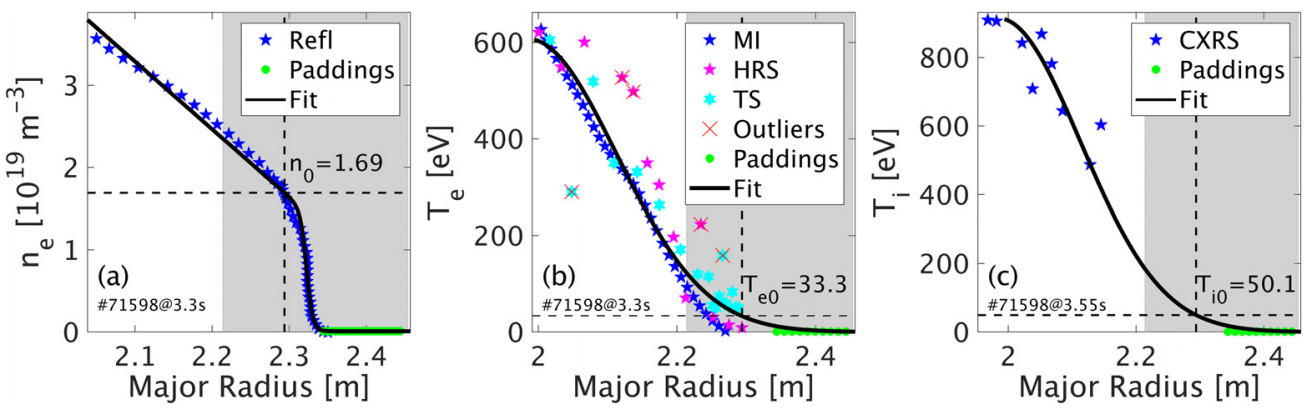

FIG. 2. An example to demonstrate the fits of the measured electron density and electron and ion temperature profiles. The red crosses indicate that the specified data are outliers, the dashed vertical lines specify the position of the LCFS, dashed horizontal lines indicate the reference values at the LCFS, and the shaded areas indicate the simulation region in the radial direction. 

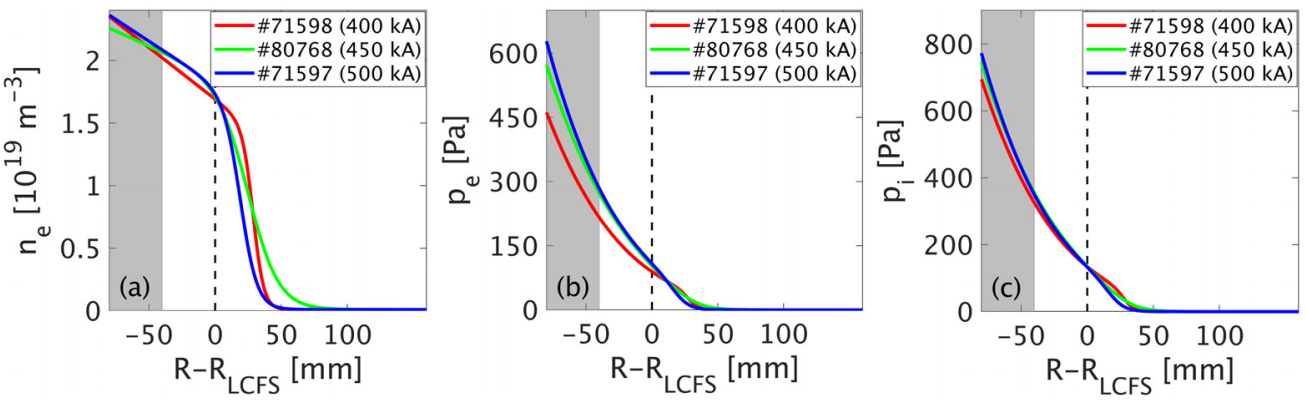

FIG. 3. The initial profiles of the electron density and the electron and ion pressures for the simulations. Dashed vertical lines specify the position of the LCFS and the shaded areas indicate the profile forcing region in the radial direction.

heuristic drift (HD)-based model. ${ }^{14}$ The amplitude of the EAST Lmode scaling is about twice that of the Eich scaling due to the broadening effects induced by the LHW heating. ${ }^{8,11}$ Although the L-mode scaling in EAST NBI-heated plasmas is reported in Ref. 8, it is evaluated at the inner divertor and the in-out asymmetry of the SOL power width in EAST is significant. ${ }^{10}$ The predictions by the HD model are evaluated by Eq. (1) in Ref. 14 with the ion drift motion (scales as $\left.\propto T_{i, L C F S}^{1 / 2} / B_{p}\right)$. The simulated $\lambda_{q}$ agrees well with the predictions by the HD model which is the same as the similar comparison in the BOUT ++ simulations, ${ }^{17}$ while it is a little bit smaller than the Eich scaling. The smaller predictions by the HD model compared with the Eich scaling are due to the lower ion temperature in L-mode plasmas. In the HD model, the magnetic drift has an effective radial component when particles stream downward to the divertor targets. However, the magnetic drift does not have any influence on the SOL power width in the HESEL simulations. Because the HESEL model is implemented in a $2 \mathrm{D}$ box, the magnetic drift is directed along the poloidal direction and has no contribution to the radial transports. Thus, the HESEL model has a different physical basis on determining the SOL power width but produces results that are relatively consistent with the Eich scaling.

\section{Numerical scaling of the SOL power width}

Taking advantage of the low computation time of the HESEL code, a numerical scaling that is dependent on the safety factor $q_{95}$, the electron temperature at the LCFS $T_{e, L C F S}$, and the electron density at the LCFS $n_{e, L C F S}$ is performed based on the low-current case (shot
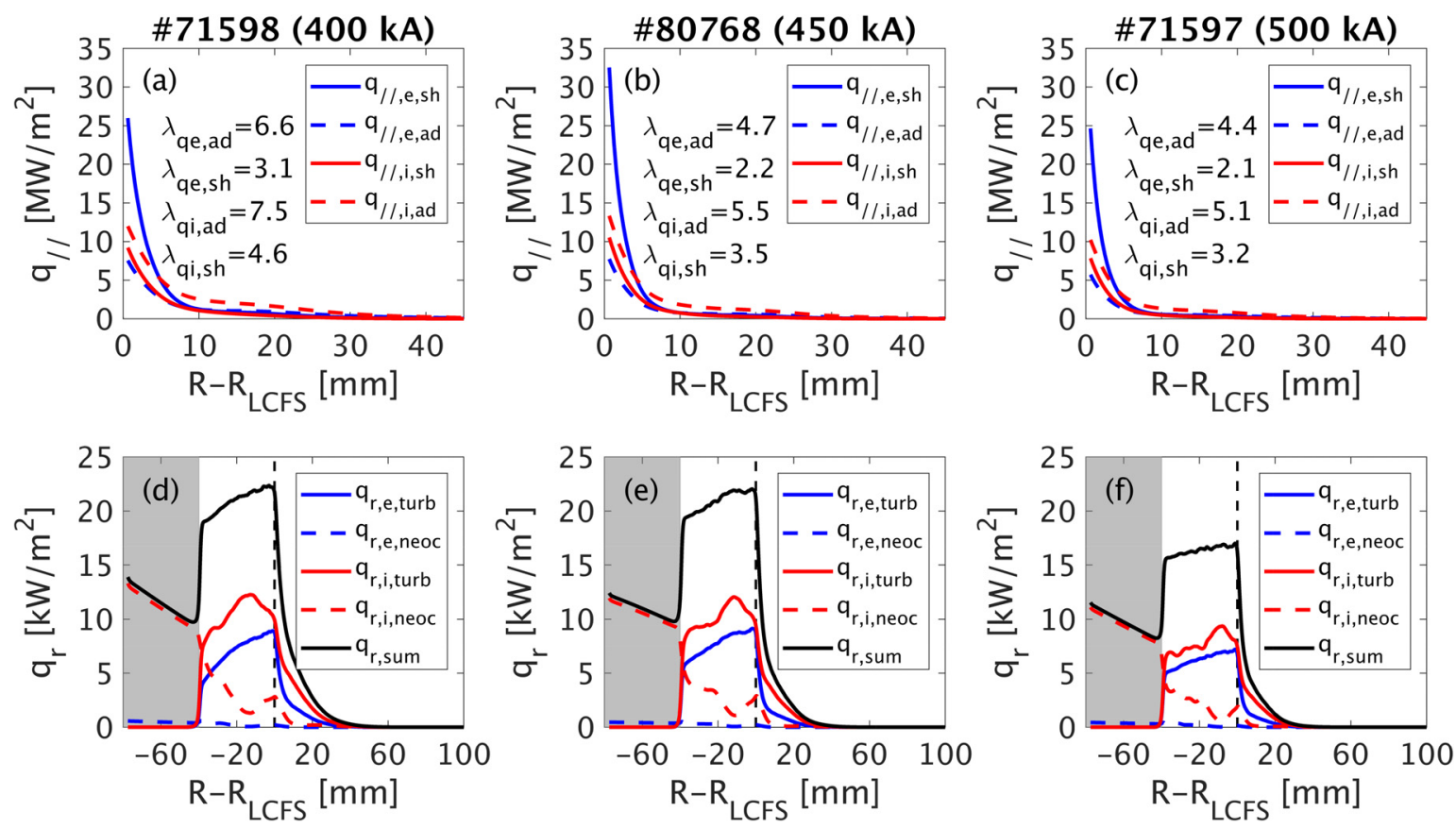

FIG. 4. The time-averaged parallel and radial heat fluxes in the saturation phase for the simulated EAST L-mode discharges. The shaded areas indicate the profile forcing region in the radial direction. The black solid lines represent the sum of all four kinds of the radial heat fluxes. 

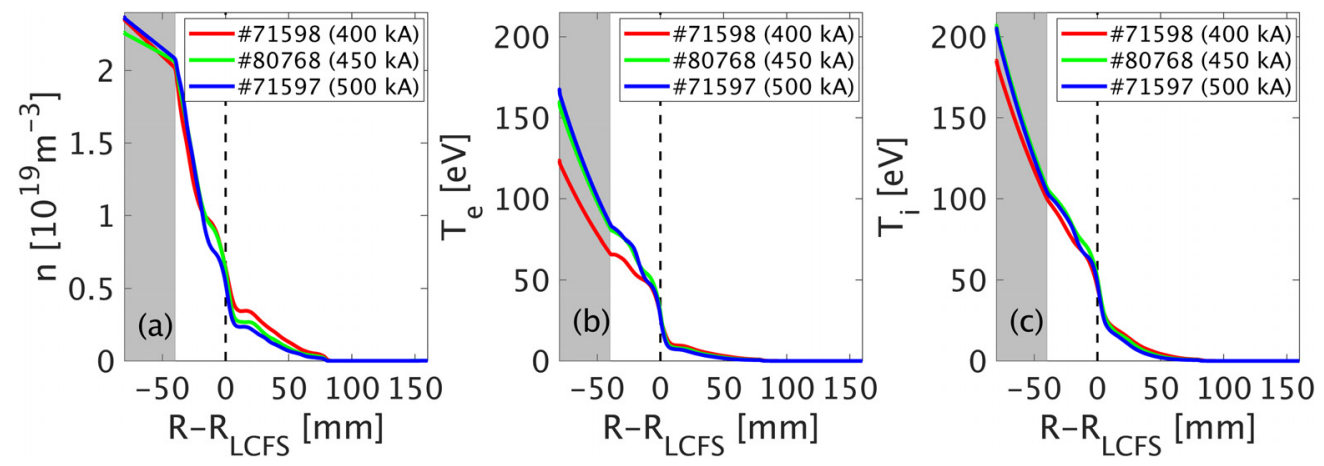

FIG. 5. The time-averaged electron density and electron and ion temperature profiles (poloidally averaged before averaging in time) in the saturation phase. The shaded areas indicate the profile forcing region in the radial direction.

\#71598) where $\lambda_{q}$ has the closest value to the Eich scaling. These parameters are scanned separately in the following ranges: $q_{95} \in[4.5$, 8.5], $T_{e, L C F S}[\mathrm{eV}] \in[10.0,50.0]$, and $n_{e, L C F S}\left[10^{19} \mathrm{~m}^{-3}\right] \in[1.0,3.1]$. For the scans of $n_{e, L C F S}$ and $T_{e, L C F S}$, the shapes of the corresponding initial profiles are kept the same and only the amplitudes of the initial profiles vary. Note that the amplitude of the initial ion temperature changes accordingly with that of the initial electron temperature to make $T_{i, L C F S} / T_{e, L C F S}$ a constant, and the grid spacing and the poloidal and radial grid sizes are kept fixed, while $T_{e, L C F S}$ is scanned.

Figure 8 shows the regression results of the power widths for the total parallel heat flux, the parallel electron heat flux, and the parallel ion heat flux. We see that all three scalings have the similar scaling dependences on $q_{95}, n_{e, L C F S}$, and $T_{e, L C F S}$. The numerical scaling of the SOL power width employing the full collisional model is then written as

$$
\lambda_{q}=(11.46 \pm 4.00) q_{95}^{1.31 \pm 0.18} T_{e, L C F S}^{-1.03 \pm 0.03} n_{e, L C F S}^{0.57 \pm 0.07} .
$$

Compared with Eq. (1), the exponent of $T_{e, L C F S}$ in Eq. (19) is more negative and the exponents of $q_{95}$ and $n_{e, L C F S}$ are larger, probably because the standard collisional model has no variations of the electron and ion temperatures and the electron density in the neoclassical diffusion coefficients. Note that the parameters in Eq. (19) are scanned individually and are independent of each other, meaning that their scaling exponents will not change much if the other scaling parameters are omitted, allowing us to compare one or more scaling parameter(s) with the experimental scalings. Since $q_{95} \propto B_{p}^{-1}$, Eq. (19) can be modified as $\lambda_{q} \propto B_{\mathrm{p}}^{-1.31} T_{e, L C F S}^{-1.03} n_{e, L C F S}^{0.57}$. The exponent of $B_{p}$ is relatively consistent with the Eich scaling $\left(\lambda_{q, \text { Eich }}=0.63 B_{\mathrm{p}}^{-1.19}\right)$. ${ }^{4}$ Compared with the AUG L-mode scaling $\left(\lambda_{q, A U G} \propto B_{\mathrm{p}}^{-0.66} T_{e, L C F S}^{-0.93} A^{-0.15}\right.$ if the stiff pressure profile is assumed, where $A$ is the mass number of the main ions), ${ }^{7}$ the exponent of $B_{p}$ is more negative and the exponent of $T_{e, L C F S}$ is similar. Normally, $T_{e, L C F S}$ has a positive correlation with $B_{p}$ in experiments, ${ }^{10}$ suggesting that $\lambda_{q, A U G}$ might have a stronger dependence on $T_{e, L C F S}$ if $B_{p}$ is omitted. More dedicated experiments should be carried out to fully examine this numerical scaling in L-mode plasmas.

\section{Interpretation of the numerical scaling}

The safety factor $q_{95}$ enters the HESEL model through the neoclassical correction factor $C_{\text {neoc,corr }}=1+q_{95}^{2} R / a$ [see Eq. (9)] and the ballooning length $L_{b} \approx q_{95} R$. To find out which dynamics (the parallel or radial heat transports) $q_{95}$ influences the most in determining the SOL power width, $C_{\text {neoc,corr }}$ and $L_{b}$ are kept fixed separately in the scan of $q_{95}$ from 4.0 to 8.0. The results of the simulated SOL power width $\lambda_{q}$ vs $q_{95}$ in these two scans are shown in Fig. 9(a). It is clear that $\lambda_{q}$ does not change much in the scan where $L_{b}$ is fixed but scales consistently with Eq. (19) in the scan where $C_{n e o c, c o r r}$ is fixed, meaning that $q_{95}$ influences the SOL power width mostly through the parallel heat transports. Since $q_{95} \propto B_{p}^{-1}$, this result indicates that the scaling dependence on the poloidal magnetic field (or the plasma current) for the SOL power width scalings is essentially the scaling dependence on the ballooning length, which might explain why simulation
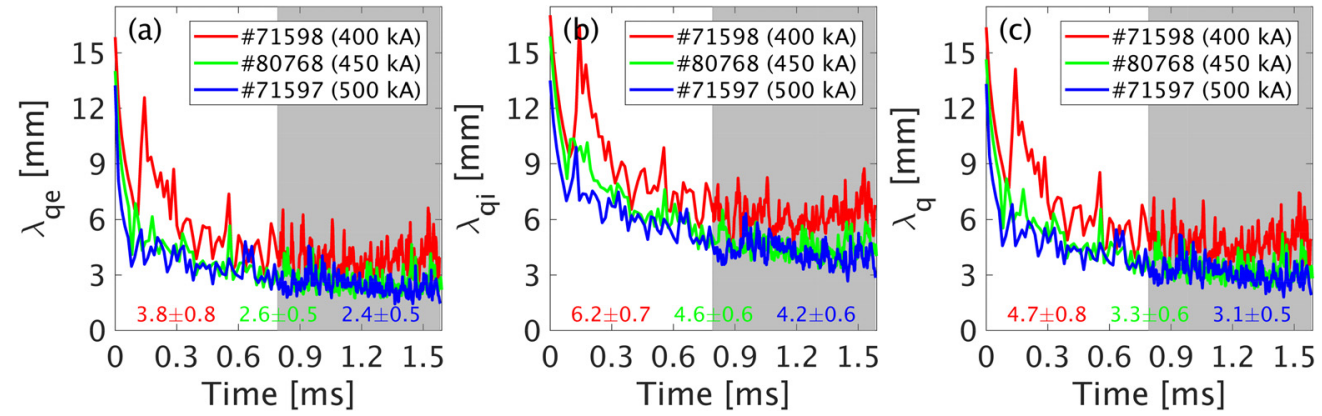

FIG. 6 . The evolution of the parallel electron heat flux width $\lambda_{q e}$, the parallel ion heat flux width $\lambda_{\text {qi }}$, and the total parallel heat flux width $\lambda_{q}$. The mean values and the standard deviations are calculated using the data in the saturation phase in the shaded areas. 


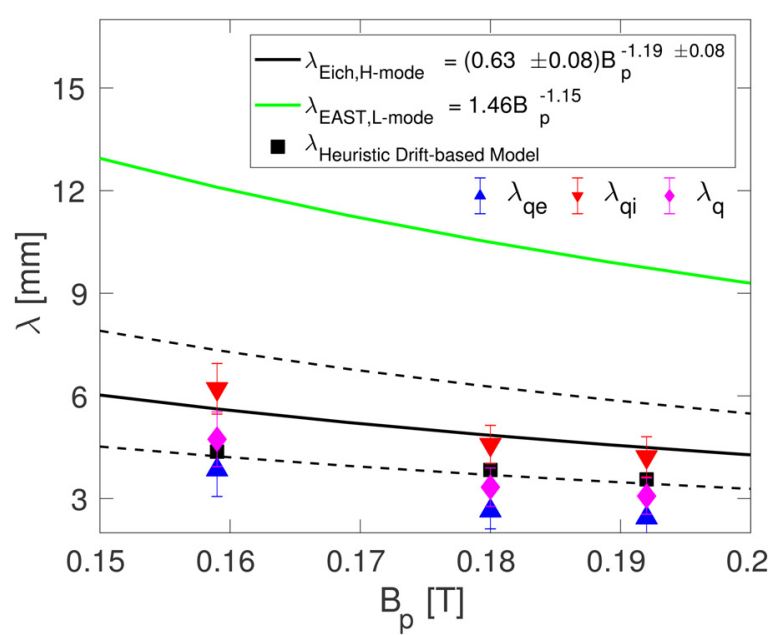

FIG. 7. The simulated SOL power widths based on EAST L-mode discharges and the comparisons with the Eich scaling (black solid line), the EAST L-mode scaling (green solid line), and the predictions by the heuristic drift-based model (black solid squares). The dashed black lines are the regression uncertainties for the Eich scaling.

codes $^{17,19,21,22}$ with different radial heat transports but similar formulation of the parallel heat transports can reproduce the inverse scaling dependence on the poloidal magnetic field. In this sense, the numerical scaling that is dependent on the ballooning length is proposed as [see also Fig. 9(b)]

$$
\lambda_{q}=(0.18 \pm 0.08) L_{b}^{1.31 \pm 0.16}
$$

The interpretation of the scaling dependence on $T_{e, L C F S}$ is much more complicated. Three cases, where $T_{e, L C F S}$ is scanned for the numerical scaling, are selected to examine the radial heat conductivity and the parallel heat damping velocities. The results are shown in Fig. 10, where the electron neoclassical heat conductivity and the parallel ion Spitzer-Härm damping velocity are neglected. According to Fig. 10 (a), we learn that the ion neoclassical heat conductivity $\chi_{r, \text { i, neoc }}$ is relatively small compared with the electron (ion) turbulent heat conductivity $\chi_{r, e, t u r b}\left(\chi_{r, i, t u r b}\right)$ and $\chi_{r, e, \text { turb }}$ is larger than $\chi_{r, i, t u r b}$ around $R-R_{L C F S}$ $=15 \mathrm{~mm}$. In Fig. $10(\mathrm{~b})$, the parallel heat advection velocity $v_{/ /, a d}$ is larger than the parallel damping velocity of the electron heat conduction $v_{/ /, e s h}$ in the far SOL region. The profile of $v_{/ /, a d}$ is also flatter than that of $v_{/ /, e, s h}$ because of their different temperature dependences. As $T_{e, L C F S}$ increases, the turbulent heat conductivity and the parallel heat damping velocities decrease and increase, respectively, leading to a narrower SOL power width.

The SOL power width is intrinsically determined by the competition between the parallel and radial heat transports. If a constant radial heat conductivity $\chi_{r}$ and a constant parallel damping velocity $v_{/ /}$are used to characterize these two processes, then the SOL power width $\lambda_{q}$ $\approx L_{b} \chi_{r} /\left(\lambda_{T} v_{/}\right)$, where $\lambda_{T}$ is the width of the temperature profile. If $\lambda_{T} /$ $\lambda_{q}$ is a constant $\gamma$, then $\lambda_{q}$ is appropriately equal to ${ }^{12}$

$$
\lambda_{q} \approx \sqrt{\frac{L_{b} \chi_{r}}{\gamma v_{/ /}}} .
$$

Using Eq. (21), we can identify which dynamics is influenced the most by $T_{e, L C F S}$. The normalized turbulent heat conductivity and the normalized reciprocal of the parallel heat damping velocities with respect to the ones where $T_{e, L C F S}=10 \mathrm{eV}$ are plotted in Fig. 11(a). Note that the heat conductivity and the damping velocities are averaged radially in the following ranges, $R-R_{L C F S}[\mathrm{~mm}] \in[-5,5]$ and $R-R_{L C F S}[\mathrm{~mm}] \in[0,5]$, respectively. As $T_{e, L C F S}$ increases, the normalized electron and ion turbulent heat conductivity decrease almost at the same pace and then seem to saturate, the normalized reciprocal of the parallel advection velocity decreases slowly and saturates quickly, but the normalized reciprocal of the parallel conduction velocity decreases and saturates even quicker than the normalized turbulent heat conductivity. So opposite of what was found for $q_{95}$ or $L_{b}, T_{e, L C F S}$ influences both the radial and parallel heat transports.

The same method used above can be applied to investigate the scaling dependence on the reference electron density $n_{e, L C F S}$ for the numerical scaling in Eq. (19). From the results shown in Fig. 11(b), the electron and ion turbulent conductivity increase significantly as $n_{e, L C F S}$ increases, while the parallel damping velocities do not change as much as the turbulent conductivity. So, the scaling dependence on $n_{e, L C F S}$ in Eq. (19) is mainly a result of the change of the radial turbulent heat transports.

\section{E. Discussions on H-mode plasmas and implication for ITER}

As mentioned in the Introduction, the power width scaling has a weaker scaling dependence on temperature for $\mathrm{H}$-mode plasmas $\left(\lambda_{q} \propto T_{e, \text { edge }}^{-0.5}\right)$ than that for L-mode plasmas $\left(\lambda_{q} \propto T_{e, e d g e}^{-0.93}\right){ }^{7,10}$ In $\mathrm{H}-$ mode plasmas, the electron-ion collisionality in the SOL is much weaker than that in L-mode plasmas. Thus, the Spitzer-Härm heat conduction is not enough to describe the parallel heat conduction in $\mathrm{H}$-mode plasmas. Normally, a combination of the Spitzer-Härm and the flux-limited heat conduction is employed to solve the issue. ${ }^{13}$ Since the parallel damping velocities for the Spitzer-Härm and the fluxlimited heat conduction are

$$
\begin{aligned}
& v_{/ /, e, s h} \propto T_{e}^{5 / 2} /\left(n_{e} L_{b}\right), \\
& v_{/ /, e, f l} \propto T_{e}^{1 / 2},
\end{aligned}
$$

respectively, the flux-limited heat conduction has a weaker temperature dependence than that for the Spitzer-Härm heat conduction. In addition, the radial turbulent heat transport is also weaker in $\mathrm{H}$-mode plasmas. So, the scaling dependence on $T_{e, L C F S}$ for the power width scaling shall be weakened in $\mathrm{H}$-mode plasmas. In order to demonstrate the influence of Spitzer-Härm heat conduction on the scaling dependence on $T_{e, L C F S}$, we rescanned $T_{e, L C F S}$ with Spitzer-Härm heat conduction being turned off (heat advection is kept and it has the same temperature dependence as that for the flux-limited heat conduction). From the scaling results shown in Fig. 12, it is clear that the scaling exponent is less negative $\left(\lambda_{q} \propto T_{e, L C F S}^{-0.39}\right)$ in the case without SpitzerHärm heat conduction, which communicates with the EAST H-mode scaling $\left(\lambda_{q} \propto T_{e, e d g e}^{-0.5} \propto T_{e, L C F S}^{-0.5}\right)$, suggesting that the weakened scaling dependence on $T_{e, \text { edge }}$ in H-mode plasma is probably due to the decrease in the electron-ion collisionality.

Interestingly, the heuristic drift-based model has a positive scaling dependence on $T_{i, L C F S}\left(\lambda_{S O L} \propto T_{i, L C F S}^{1 / 2} / B_{p}\right)$ that is different from the negative scaling dependence on $T_{e, L C F S}$ in the numerical 

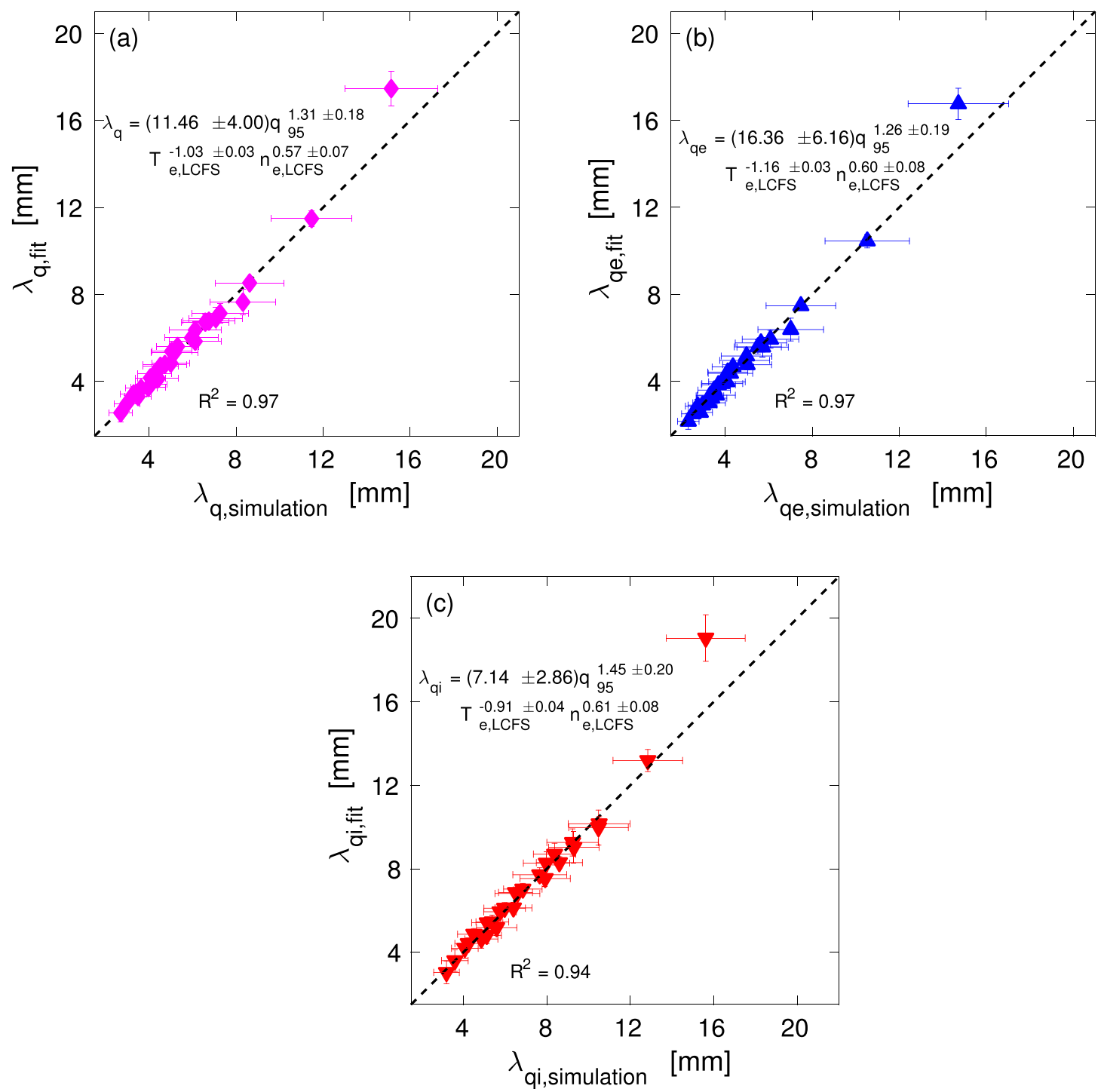

FIG. 8. The numerical scalings of the SOL power widths with respect to the safety factor $q_{95}$, the reference electron temperature at the LCFS $T_{e, L C F S}$, and the reference electron density at the LCFS $n_{e, L C F S}$ for (a) the total parallel heat flux, (b) the parallel electron heat flux, and (c) the parallel ion heat flux.

scalings in Fig. 8 ( $T_{i, L C F S} / T_{e, L C F S}$ is a constant in the scan of $T_{e, L C F S}$ ) but gives consistent results with the HESEL code (see Fig. 7). This might indicate that the scaling dependence on $T_{e, L C F S}$ is not as significant as the scaling dependence on $B_{p}$ or $q_{95}$, meaning that the parallel heat transports is more important in determining the SOL power width as mentioned in Subsection IV D. Based on this idea, we could use the numerical scaling in Eq. (20) to make prediction for ITER. Since the major radius $R$ and the toroidal magnetic field at the magnetic axis $B_{t}$ influence the interchange instability which dominates the radial heat transport in the HESEL model, a modified scaling is obtained by scanning additional parameters $R$ (the aspect ratio is kept constant) and $B_{t}$ separately in the following ranges: $R[\mathrm{~m}] \in[1.0,6.5], B_{t}[\mathrm{~T}] \in[1.0,6.0]$, which is

$$
\begin{aligned}
\lambda_{q} & =(0.26 \pm 0.16) q_{95}^{1.30 \pm 0.32} B_{t}^{-0.33 \pm 0.10} R^{1.32 \pm 0.04} \\
& =(0.23 \pm 0.03) B_{t}^{-0.29 \pm 0.09} L_{b}^{1.33 \pm 0.03} .
\end{aligned}
$$

Clearly, except for the already known scaling dependence on the ballooning length in Eq. (23), which is supported by the recent result in TCV $\left(\lambda_{q, T C V} \propto L_{/ \mid}^{0.92}\right)$ where the length of the outer divertor leg was scanned, ${ }^{34} \lambda_{q}$ also has a weak negative dependence on $B_{t}$, which probably results from the presence of $B_{t}$ in $\mathrm{E} \times \mathrm{B}$ drift terms $(\{\varphi, f\} / B)$. Compared with the Eich scaling $\left(\lambda_{q} \propto q_{95}^{1.1} B_{t}^{-0.8} R^{0}\right),{ }^{4}$ Eq. (23) has a similar exponent of $q_{95}$, a less negative exponent of $B_{t}$, but a much larger exponent of $R$. This difference, especially the stronger scaling dependence on $R$, leads to a very different prediction for ITER. For the ITER baseline H-mode scenario $\left(q_{95}=3.0, B_{t}=5.3, R=6.2\right)$, 

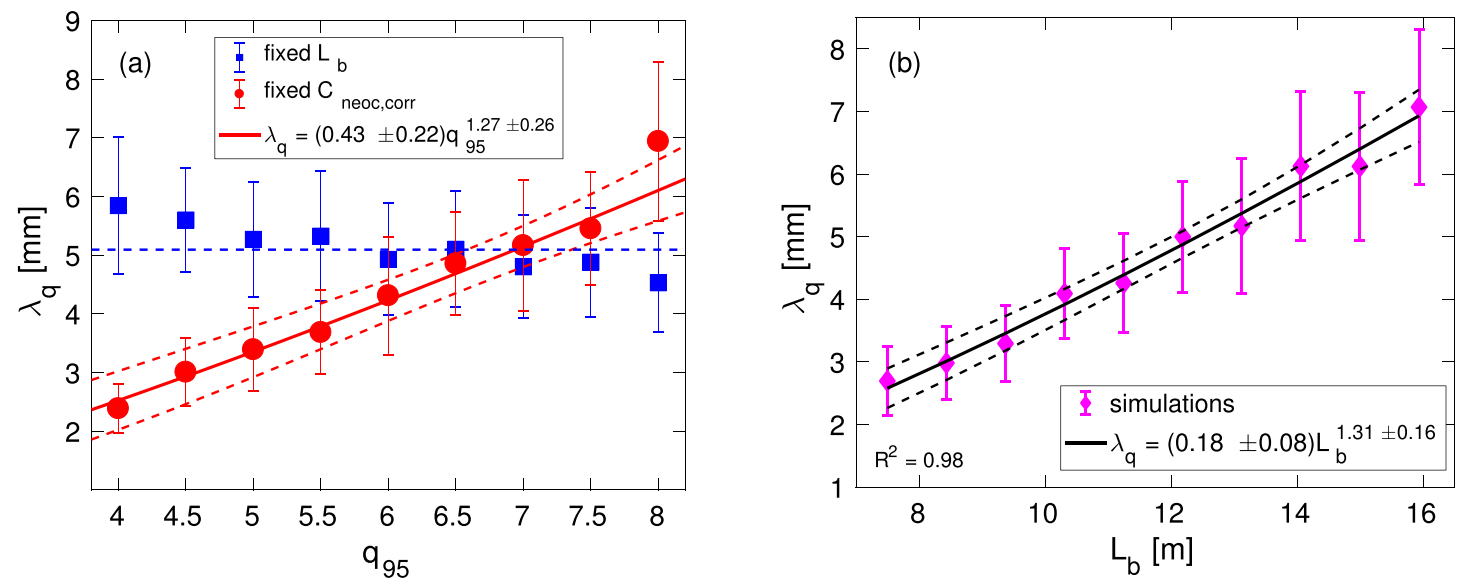

FIG. 9. (a) The results of the simulated SOL power width $\lambda_{q}$ vs the safety factor $q_{95}$ in two scans of $q_{95}$ where the neoclassical correction factor $C_{\text {neoc,corr }}$ and the ballooning length $L_{b}$ are kept fixed separately. The red solid and dashed lines are regression result and uncertainties for the scan where $C_{\text {neoc,corr }}$ is fixed. (b) The numerical scaling of $\lambda_{a}$ with respect to $L_{b}$.
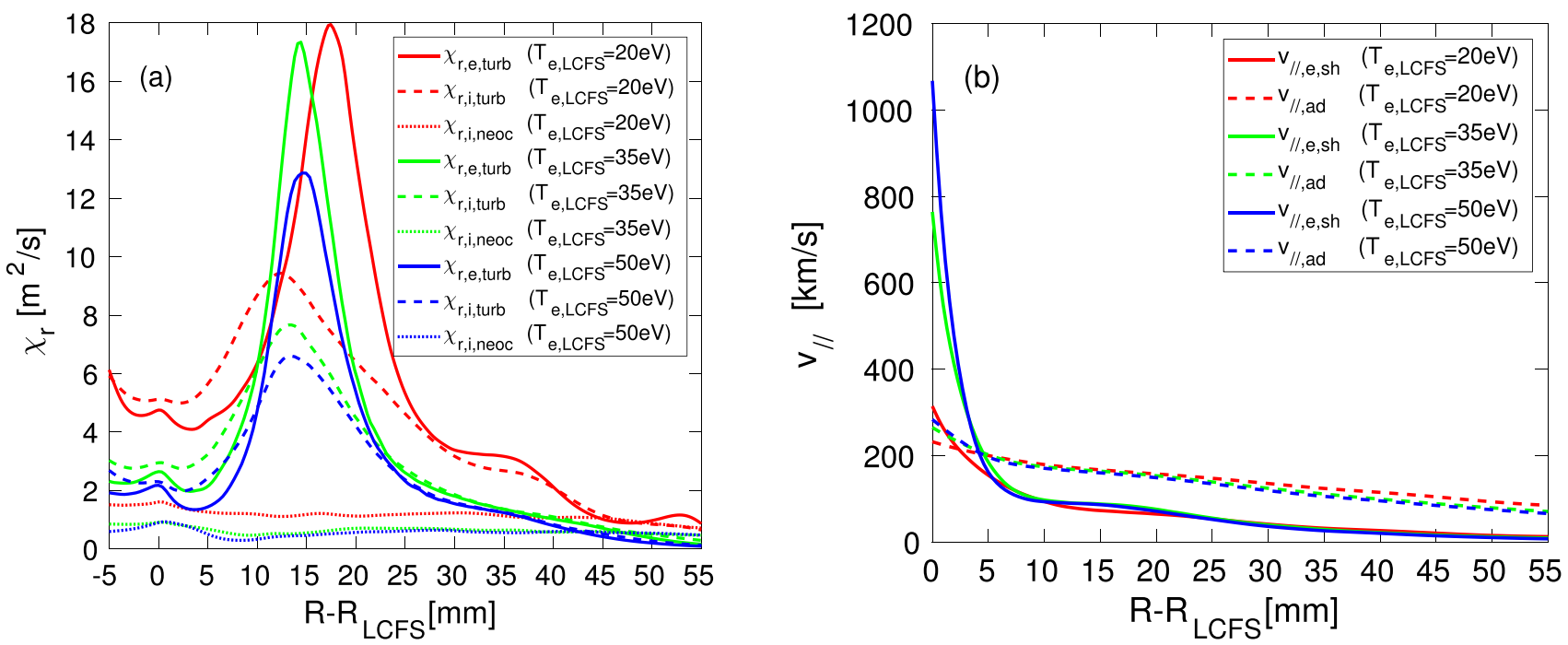

FIG. 10. (a) The radial heat conductivity and (b) the parallel heat damping velocities of three selected cases where $T_{e, L C F S}$ is scanned for the numerical scaling.

Eq. (23) predicts $\lambda_{q, I T E R}=6.95 \mathrm{~mm}$. Considering that the plasma temperature and density are much larger than those in shot \#71598 (see Table I), this prediction is probably overestimated but communicates with the prediction by the gyro-kinetic XGC1 code $\left(\lambda_{q, \text { ITER }}\right.$ $=5.9 \mathrm{~mm}){ }^{19}$ Since the scaling dependence on the major radius is extremely important for the interpolation to ITER, the multimachine databases must be carefully examined and the dedicated crossmachine experiments have to be conducted to confirm this scaling dependence. In principle, Eq. (23) has to be validated and includes $n_{e, L C F S}$ and $T_{e, L C F S}$ in H-mode plasma. Unfortunately, current HESEL code cannot be used to simulate $\mathrm{H}$-mode plasmas due to the numerical instability, which stops the simulation when diffusion coefficients in Eq. (9) are small, and insufficient physics to maintain the H-mode pedestal.

Another issue that might influence the simulation results is the ionization of the neutrals in the SOL, which cools down the plasma temperature and increases the plasma density. In L-mode plasmas with high collisionality, the electron Spitzer-Härm heat conduction dominates the parallel heat flux. If the ionization mainly happens near the LCFS, then the heat conduction will be reduced. The hotter the plasma and the denser the neutrals, the stronger the reduction of the heat conduction, which flattens the profile of the parallel heat flux, 

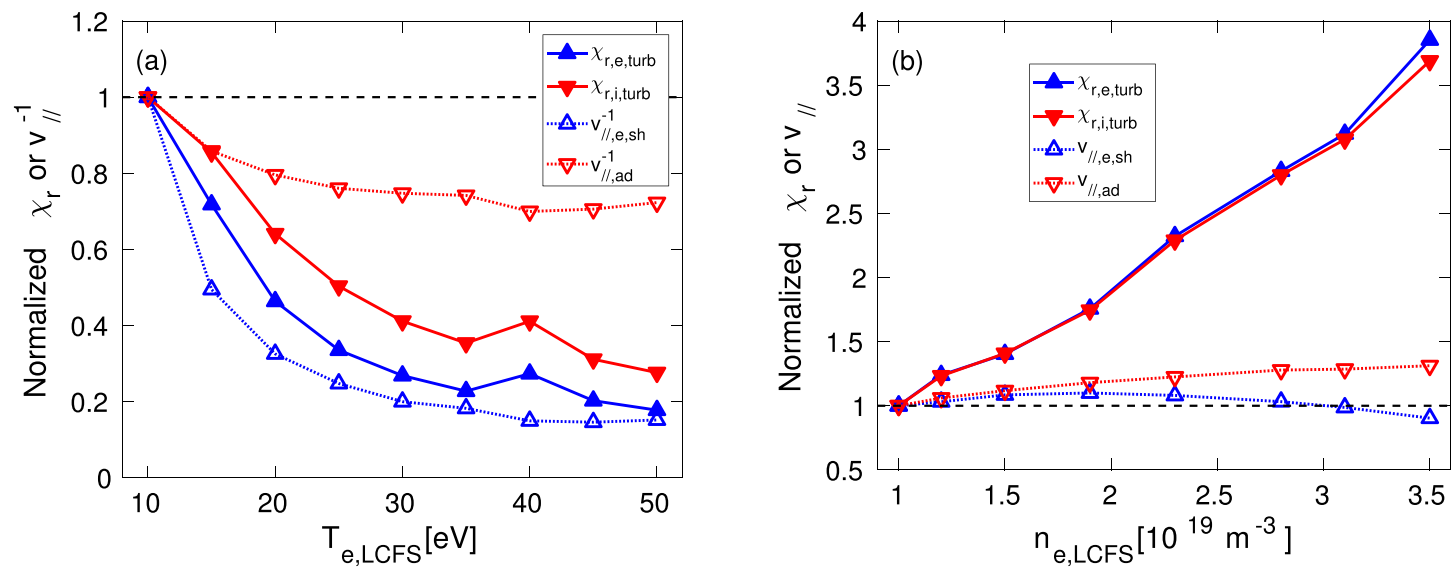

FIG. 11. The comparisons of the dependences on (a) the reference electron temperature $T_{e, L C F S}$ and (b) the reference electron density $n_{e, L C F S}$ for the radial turbulent heat conductivity and the parallel heat damping velocities. All the data are normalized with respect to the data where (a) $T_{e, L C F S}=10 \mathrm{eV}$ and $(\mathrm{b}) n_{e, L C F S}=1.0 \times 10^{19} \mathrm{~m}^{-3}$.

leading to a larger SOL power width. If the ionization is far away from the LCFS, we expect that the influence on the SOL power width is limited. A detailed study of neutrals' impact on the simulation results will be investigated in the future work.

\section{CONCLUSIONS}

In this paper, the SOL power width scaling has been studied by the newly reimplemented HESEL code based on dedicatedly selected EAST L-mode discharges. The analysis of the components of the parallel and radial heat fluxes for the simulations shows that the electron and ion turbulent heat fluxes dominate the radial heat transports, and the Spitzer-Härm heat conduction and the heat advection dominate the parallel electron and ion heat transports, respectively. The simulated SOL power width is consistent with the heuristic drift-based model and the Eich scaling.

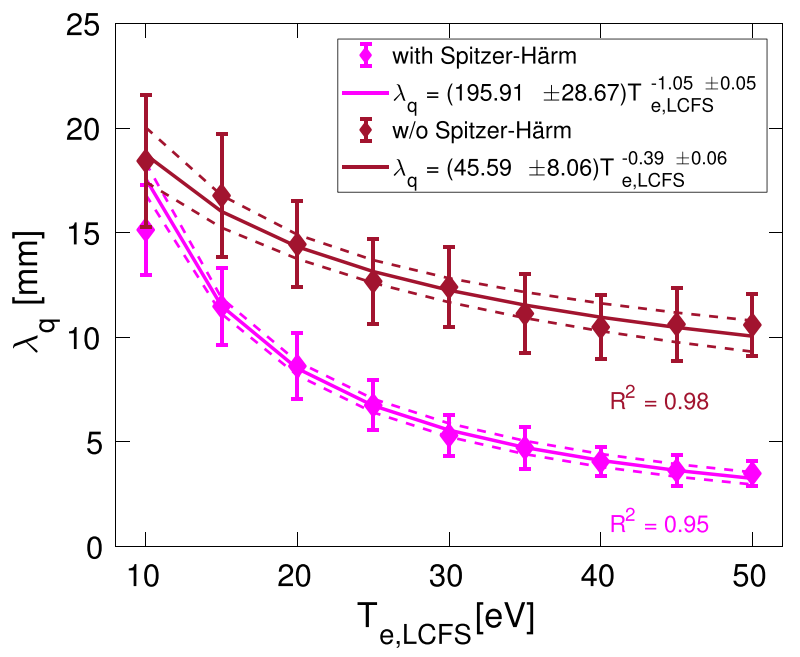

FIG. 12. The scaling dependence of the simulated SOL power widths on the reference electron temperature $T_{e, L C F S}$. (a) With Spitzer-Härm heat conduction and (b) without Spitzer-Härm heat conduction.
A numerical scaling has been performed and the result $\left(\lambda_{q}=11.46 q_{95}^{1.31} T_{e, L C F S}^{-1.03} n_{e, L C F S}^{0.57} \propto B_{\mathrm{p}}^{-1.31} T_{e, L C F S}^{-1.03} n_{e, L C F S}^{0.57}\right) \quad$ communicates with the Eich scaling $\left(\lambda_{q, \text { Eich }}=0.63 B_{\mathrm{p}}^{-1.19}\right)^{4}$ and the AUG L-mode scaling $\left(\lambda_{q, A U G} \propto B_{\mathrm{p}}^{-0.66} T_{e, L C F S}^{-0.93} A^{-0.15}\right)$. $^{\mathrm{p}}$ A further investigation on the numerical scaling reveals that the safety factor influences the SOL power width mainly through the parallel heat transports and the scaling dependence on the poloidal magnetic field (or the plasma current) for the experimental scalings is most likely the scaling dependence on the ballooning length, $q_{95} R$. The edge electron density influences the SOL power width mainly through the radial turbulent heat transports, while the edge electron temperature influences the SOL power width through both the parallel and radial heat transports.

A further numerical scaling that is dependent on the engineering parameters, $\lambda_{q}=0.26 q_{95}^{1.30} B_{t}^{-0.33} R^{1.32}=0.23 B_{t}^{-0.29} L_{b}^{1.33}$, predicts $\lambda_{q, I T E R}=6.95 \mathrm{~mm}$ for the ITER baseline H-mode scenario, which is much larger than the prediction by the Eich scaling $\left(\lambda_{q, I T E R} \approx 1 \mathrm{~mm}\right)$ but communicates with the prediction by the gyro-kinetic XGC1 code $\left(\lambda_{q, I T E R}=5.9 \mathrm{~mm}\right)$. Since the scaling dependence on the major radius is extremely important for the interpolation to ITER, the multimachine databases must be carefully examined, the dedicated cross-machine experiments should be conducted, and this numerical scaling also have to be validated in $\mathrm{H}$-mode plasmas to confirm this strong scaling dependence on the major radius in the numerical scaling.

\section{ACKNOWLEDGMENTS}

The author thanks Benjamin Dudson for the help to diagnose issues on implementing the HESEL model with the BOUT ++ framework. This work was supported by the National Key Research and Development Program (Nos. 2016YFA0400600, 2017YFE0301300, and 2017YFA0402500), China Scholarship Council (No. 201704910761), National Natural Science Foundation of China (No. 11575236), and National Magnetic Confinement Fusion Science Program of China (Nos. 2015GB101000 and 2015GB103000). This work was also partly supported by the Science Foundation of Institute of Plasma Physics Chinese Academy of Sciences (No. DSJJ-18-02), AHNSF (No. 1808085J07), Users with Excellence Project of Hefei Science Center CAS (No. 2018HSC-UE008). 


\section{REFERENCES}

${ }^{1}$ A. Loarte, S. Bosch, A. Chankin, S. Clement, A. Herrmann, D. Hill, K. Itami, J. Lingertat, B. Lipschultz, K. McCormick, R. Monk, G. D. Porter, M. Shimada, and M. Sugihara, J. Nucl. Mater. 266-269, 587-592 (1999).

${ }^{2}$ T. Eich, B. Sieglin, A. Scarabosio, W. Fundamenski, R. J. Goldston, A. Herrmann, and ASDEX Upgrade Team, Phys. Rev. Lett. 107, 215001 (2011).

${ }^{3}$ M. A. Makowski, D. Elder, T. K. Gray, B. LaBombard, C. J. Lasnier, A. W. Leonard, R. Maingi, T. H. Osborne, P. C. Stangeby, J. L. Terry, and J. Watkins, Phys. Plasmas 19, 056122 (2012).

${ }^{4}$ T. Eich, A. W. Leonard, R. A. Pitts, W. Fundamenski, R. J. Goldston, T. K. Gray, A. Herrmann, A. Kirk, A. Kallenbach, O. Kardaun, A. S. Kukushkin, B. LaBombard, R. Maingi, M. A. Makowski, A. Scarabosio, B. Sieglin, J. Terry, A. Thornton, ASDEX Upgrade Team, and JET EFDA Contributors, Nucl. Fusion 53, 093031 (2013).

${ }^{5}$ L. Wang, H. Y. Guo, G. S. Xu, S. C. Liu, K. F. Gan, H. Q. Wang, X. Z. Gong, Y. Liang, X. L. Zou, J. S. Hu, L. Chen, J. C. Xu, J. B. Liu, N. Yan, W. Zhang, R. Chen, L. M. Shao, S. Ding, G. H. Hu, W. Feng, N. Zhao, L. Y. Xiang, Y. L. Liu, Y. L. Li, C. F. Sang, J. Z. Sun, D. Z. Wang, H. B. Ding, G. N. Luo, J. L. Chen, X. Gao, L. Q. Hu, B. N. Wan, J. Li, and EAST Team, Nucl. Fusion 54, 114002 (2014).

${ }^{6}$ J. B. Liu, H. Y. Guo, L. Wang, G. S. Xu, H. Q. Wang, S. C. Liu, W. Feng, J. C. $\mathrm{Xu}, \mathrm{G}$. Z. Deng, Q. Q. Yang, and B. L. Ling, Fusion Eng. Des. 100, 301-306 (2015).

${ }^{7}$ B. Sieglin, T. Eich, M. Faitsch, A. Herrmann, A. Scarabosio, and ASDEX Upgrade Team, Plasma Phys. Controlled Fusion 58, 055015 (2016).

${ }^{8}$ G. Z. Deng, J. C. Xu, X. Liu, X. J. Liu, J. B. Liu, H. Zhang, S. C. Liu, L. Chen, N. Yan, W. Feng, H. Liu, T. Y. Xia, B. Zhang, L. M. Shao, T. F. Ming, G. S. Xu, H. Y. Guo, X. Q. Xu, X. Gao, and L. Wang, Plasma Phys. Controlled Fusion 60, 045001 (2018).

${ }^{9}$ D. Brunner, B. LaBombard, A. Q. Kuang, and J. L. Terry, Nucl. Fusion 58, 094002 (2018).

${ }^{10}$ X. Liu, V. Naulin, J. C. Xu, G. Z. Deng, J. B. Liu, J. J. Rasmussen, A. H. Nielsen, R. Goldston, R. Maingi, Y. Liu, G. S. Xu, L. Wang, J. Li, and EAST Team, Plasma Phys. Controlled Fusion 61, 045001 (2019).

${ }^{11}$ L. Wang, M. A. Makowski, H. Y. Guo, A. W. Leonard, G. S. Xu, X. Z. Gong, A. M. Garofalo, G. Z. Deng, T. W. Petrie, D. Thomas, G. Jackson, G. Kramer, R. Maingi, J. B. Liu, W. Feng, H. Wang, B. Zhang, S. Ding, C. K. Pan, Y. J. Hu, J. P. Qian, J. Li, and B. N. Wan, Nucl. Mater. Energy 12, 221-226 (2017).

${ }^{12}$ J. W. Connor, G. F. Counsell, S. K. Erents, S. J. Fielding, B. LaBombard, and K. Morel, Nucl. Fusion 39(2), 169 (1999).

${ }^{13}$ P. C. Stangeby, J. M. Canik, and D. G. Whyte, Nucl. Fusion 50, 125003 (2010).

${ }^{14}$ R. J. Goldston, Nucl. Fusion 52, 013009 (2012).

${ }^{15} \mathrm{X}$. Q. Xu, B. Dudson, P. B. Snyder, M. V. Umansky, and H. Wilson, Phys. Rev. Lett. 105, 175005 (2010).

${ }^{16}$ B. D. Dudson, M. V. Umansky, X. Q. Xu, P. B. Snyderc, and H. R. Wilson, Comput. Phys. Commun. 180, 1467-1480 (2009).
${ }^{17}$ T. Y. Xia, X. Q. Xu, Y. B. Wu, Y. Q. Huang, L. Wang, Z. Zheng, J. B. Liu, Q. Zang, Y. Y. Li, D. Zhao, and EAST Team, Nucl. Fusion 57, 116016 (2017).

${ }^{18}$ B. Chen, X. Q. Xu, T. Y. Xia, M. Porkolab, E. Edlund, B. LaBombard, J. Terry, J. W. Hughes, S. F. Mao, M. Y. Ye, and Y. X. Wan, Nucl. Fusion 57, 116025 (2017).

${ }^{19}$ C. S. Chang, S. Ku, A. Loarte, V. Parail, F. Köch, M. Romanelli, R. Maingi, J.W. Ahn, T. Gray, J. Hughes, B. LaBombard, T. Leonard, M. Makowski, and J. Terry, Nucl. Fusion 57, 116023 (2017).

${ }^{20}$ E. T. Meier, R. J. Goldston, E. G. Kaveeva, M. A. Makowski, S. Mordijck, V. A. Rozhansky, I. Yu Senichenkov, and S. P. Voskoboynikov, Plasma Phys. Controlled Fusion 58, 125012 (2016).

${ }^{21}$ G. Z. Deng, X. J. Liu, L. Wang, S. C. Liu, L. Chen, H. Zhang, J. C. Xu, W. Feng, J. B. Liu, H. Liu, and X. Gao, Phys. Plasmas 24, 042508 (2017).

${ }^{22}$ J. Olsen, A. H. Nielsen, J. J. Rasmussen, J. Madsen, T. Eich, B. Sieglin, and V. Naulin, Plasma Phys. Controlled Fusion 60, 085018 (2018).

${ }^{23}$ J. Madsen, V. Naulin, A. H. Nielsen, and J. J. Rasmussen, Phys. Plasmas 23, 032306 (2016).

${ }^{24}$ A. H. Nielsen, J. J. Rasmussen, J. Madsen, G. S. Xu, V. Naulin, J. M. B. Olsen, M. Løiten, S. K. Hansen, N. Yan, L. Tophøj, and B. N. Wan, Plasma Phys. Controlled Fusion 59, 025012 (2017).

${ }^{25}$ W. Fundamenski, O. E. Garcia, V. Naulin, R. A. Pitts, A. H. Nielsen, J. J. Rasmussen, J. Horacek, J. P. Graves, and JET EFDA Contributors, Nucl. Fusion 47, 417-433 (2007)

${ }^{26}$ O. E. Garcia, V. Naulin, A. H. Nielsen, and J. Juul Rasmussen, Phys. Plasmas 12, 62309 (2005).

${ }^{27}$ S. B. Zhang, X. Gao, B. L. Ling, Y. M. Wang, T. Zhang, X. Han, Z. X. Liu, J. L. Bu, J. Li, and EAST Team, Plasma Sci. Technol. 16(4), 311 (2014).

${ }^{28}$ Y. M. Wang, X. Gao, B. L. Ling, S. B. Zhang, T. Zhang, X. Han, S. C. Liu, Z. X. Liu, Y. Liu, A. Ti, and EAST Team, Fusion Eng. Des. 88, 2950-2955 (2013).

${ }^{29}$ H. Qu, T. Zhang, S. B. Zhang, F. Wen, Y. M. Wang, D. F. Kong, X. Han, Y. Yang, Y. Gao, C. B. Huang, J. Q. Cai, X. Gao, and EAST Team, Plasma Sci. Technol. 17(12), 985 (2015).

${ }^{30}$ Y. Liu, S. Schmuck, H. L. Zhao, J. Fessey, P. Trimble, X. Liu, Z. Y. Zhu, Q. Zang, and L. Q. Hu, Plasma Sci. Technol. 18(12), 1148 (2016).

${ }^{31}$ X. Liu, H. L. Zhao, Y. Liu, E. Z. Li, X. Han, C. W. Domier, N. C. Luhmann, A. Ti, L. Q. Hu, and X. D. Zhang, Rev. Sci. Instrum. 85, 093508 (2014).

${ }^{32}$ Q. Zang, J. Y. Zhao, L. Yang, Q. S. Hu, X. Q. Xi, X. X. Dai, J. H. Yang, X. F. Han, M. T. Li, and C. L. Hsieh, Rev. Sci. Instrum. 82, 063502 (2011).

${ }^{33}$ Y. Y. Li, J. Fu, B. Lyu, X. W. Du, C. Y. Li, Y. Zhang, X. H. Yin, Y. Yu, Q. P. Wang, M. von Hellermann, Y. J. Shi, M. Y. Ye, and B. N. Wan, Rev. Sci. Instrum. 85, 11E428 (2014).

${ }^{34}$ R. Maurizio, S. Elmore, N. Fedorczak, A. Gallo, H. Reimerdes, B. Labit, C. Theiler, C. K. Tsui, W. A. J. Vijvers, TCV Team, and MST1 Team Maurizio, Nucl. Fusion 58, 016052 (2018).

${ }^{35}$ A. C. C. Sips, "For the steady state operation, and the transport physics topical groups of the international tokamak physics activity," Plasma Phys. Controlled Fusion 47, A19-A40 (2005). 